\title{
FINITELY GENERATED CLASSES OF SETS OF NATURAL NUMBERS
}

\author{
JULIA ROBINSON
}

We say that a set $S$ of natural numbers is generated by a class $\mathcal{F}$ of functions if $S$ is the range of a function $F$ obtained by composition from the functions of $\mathfrak{F}$. Here we consider the identity function $I$ to be obtained by the empty composition. We consider only the case in which $\mathcal{F}$ consists of functions of one variable on and to the set of natural numbers $N$. All sets considered are subsets of $N$.

A class $\mathcal{C}$ of sets is generated by $\mathfrak{F}$ if every nonempty set of $\mathfrak{C}$ is generated by $\mathcal{F}$ and every set generated by $\mathfrak{F}$ is in $\mathcal{C}$. $\mathcal{C}$ is finitely generated if there is a finite class of functions $\mathfrak{F}$ which generates $\mathcal{C}$.

A function $F$ is compatible with $\mathcal{C}$ if $F(S)$ belongs to $\mathcal{C}$ for every $S$ in $\mathcal{C}$.

Clearly, if $\mathfrak{F}$ generates $\mathcal{C}$ then every $F$ in $\mathfrak{F}$ is compatible with $\mathcal{C}$. Also $N$ belongs to $\mathcal{C}$. Furthermore to show that $\mathfrak{F}$ generates $\mathcal{C}$ it is sufficient to prove that every $F \in \mathfrak{F}$ is compatible with $\mathfrak{C}, N \in \mathfrak{C}$, and every nonempty set in $\mathcal{C}$ is generated by $\mathfrak{F}$.

In [1] finite sets of relatively simple functions are given which generate the classes of recursively enumerable sets and diophantine sets. Here we ask: What classes of sets can be finitely generated? In particular, we will show that if $\mathcal{C}$ is a denumerable field of sets such that all finite sets belong to $\mathcal{C}$ and at least one infinite set having an infinite complement belongs to $\mathfrak{C}$, then $\mathfrak{C}$ is finitely generated.

Lemma 1. If $J$ is an infinite class of infinite sets then there is a set $M$ such that $M \cap T$ and $\bar{M} \cap T$ are infinite for every $T$ belonging to $J$.

Proof. Let each $T$ in $J$ be listed infinitely of ten in the sequence $T_{0}, T_{1}, \cdots$. We will construct $M$ and $\bar{M}$ simultaneously. At the $n$th step put the least unassigned number of $T_{n}$ in $M$ and then the least remaining unassigned number of $T_{n}$ in $\bar{M}$.

Lemмa 2. Given infinite sets $T_{0}, T_{1}, \cdots$ and nonempty sets $\boldsymbol{S}_{0}$, $S_{1}, \cdots$ such that $S_{k}=S_{l}$ whenever $T_{k} \cap T_{l}$ is not empty. Then there is a function $F$ such that $F\left(T_{n}\right)=S_{n}$.

Proof. Make a list of natural numbers such that each number occurs infinitely of ten. We will use the list to define $F$ at one argument for each step of the construction. Namely, if at a given step $n$ is the

Received by the editors April 29, 1968. 
next number in the list and if $x$ is the least unassigned number in $T_{n}$ and $y$ is the least number in $S_{n}$ not yet in $F\left(T_{n}\right)$, put $F x=y$; while if there is no such $y$, put $F x=$ the least number of $S_{n}$. Finally, in case $U T_{k} \neq N$, put $F x=0$ for every $x$ not in any $T_{k}$.

Theorem 1. Hypotheses. (1) $e$ is a denumerable class of sets. (2) If $\boldsymbol{A}$ and $B$ belong to $\mathcal{C}$ then $A \cup B$ belongs to $\mathcal{C}$. (3) $N$ belongs to $\mathcal{C}$. (4) Either (i) $\mathcal{C}$ contains all finite sets or (ii) $\mathfrak{C}$ contains only infinite sets and C contains the set obtained from a member set by adjoining a single element. (5) There is an infinite partition $\odot \subset \mathcal{C}$ of $N$ into infinite sets all of which are generated by a finite class $\mathfrak{F}$ of functions compatible with e.

Conclusion. e is finitely generated.

Proof. Let $\mathcal{P}=\left\{P_{0}, P_{1}, \cdots\right\}$. We say that $I$ is the index set of a set $S$ if $I=\left\{n: S \cap P_{n} \neq \varnothing\right\}$. Let $M$ be the set given by Lemma 1 such that every infinite index set of a set belonging to $\mathfrak{e}$ contains infinitely many elements of both $M$ and $\bar{M}$. (Concerning $\bar{M}$, we shall use only that $\bar{M}$ is infinite.) List the nonempty sets of $\mathcal{C}$ in a sequence $T_{0}$, $T_{1}, \cdots$ so that $T_{m}=N$ for every $m$ in $M$. We will define $F$ in such a way that (a) $F\left(T_{n} \cap P_{k}\right)=T_{k}$ whenever $T_{n} \cap P_{k}$ is infinite, and (b) $F\left(T_{n}\right)=N$ whenever $T_{n}$ has an infinite index set. Let us check that this is sufficient. In the first place, every nonempty set of $\mathfrak{C}$ will be generated by $\{F\} \cup \mathfrak{F}$ since $F\left(P_{k}\right)=T_{k}$. Also $F$ is compatible with $\mathfrak{C}$, i.e. for every $n, F\left(T_{n}\right)$ belongs to $\mathcal{C}$. To see this, let $I$ be the index set of $T_{n}$. If $I$ is infinite then $F\left(T_{n}\right)=N$ and $N$ belongs to $\mathcal{C}$ by (3). If $I$ is finite, then

$$
F\left(T_{n}\right)=\bigcup_{k \in I} F\left(T_{n} \cap P_{k}\right),
$$

where $F\left(T_{n} \cap P_{k}\right)$ is either finite or $\boldsymbol{T}_{k}$ according as $T_{n} \cap \boldsymbol{P}_{k}$ is finite or infinite. Hence if $\mathcal{e}$ contains all finite sets then $F\left(T_{n}\right)$ is a nonempty finite union of sets of $\mathfrak{C}$ and so $F\left(T_{n}\right)$ belongs to $\mathfrak{C}$. If $\mathfrak{C}$ consists only of infinite sets, then $T_{n} \cap P_{k}$ is infinite for some $k$. Thus $F\left(T_{n}\right)$ is the union of a nonempty finite union of sets of $\mathcal{C}$ and a finite set. Hence $F\left(T_{n}\right)$ belongs to $\mathrm{e}$.

Therefore to complete the proof, we need only define $F$ satisfying (a) and (b). Now make a list of pairs $(n, k)$ so that each pair occurs infinitely of ten. We will use this list to index the steps in the definition of $F$. At a step corresponding to $(n, k)$, if there is a number in $T_{n} \cap P_{k}$ at which $F$ has not yet been assigned, let the least such number be $x$. Put $F x$ equal to the least number in $T_{k}$ which is not already in $F\left(T_{n} \cap P_{k}\right)$ if there is such a number; otherwise put $F x$ equal to the 
least number in $T_{k}$. Also if the index set of $T_{n}$ is infinite and $y$ is the least number unassigned in $T_{n} \cap P_{m}$ for any $m$ in $M$, define $F y=k$. Then $F$ will satisfy both (a) and (b).

EXAmples. The classes of recursive sets, recursively enumerable sets, arithmetical sets, hyperarithmetical sets, are all finitely generated. We can let $P_{k}=(S D)^{k} D(\boldsymbol{N})$ since $S, D$ are compatible with each of these classes. Here $S$ is the successor function and $D$ the double function.

By the usual diagonal argument it is easy to see that the class of arithmetical sets cannot be generated by a finite class of arithmetical functions. (Similarly hyperarithmetical sets cannot be generated by a finite class of hyperarithmetical functions.) I do not know if the class of recursive sets can be generated by a finite number of recursive functions.

An interesting class of sets which is not covered by Theorem 1 is the class of polynomial sets and I do not know whether it is finitely generated. By a polynomial set, I mean the range of a polynomial with positive integer coefficients and any number of variables.

If we assume that $\mathcal{C}$ is a field of sets which contains all finite sets, then condition (5) in Theorem 1 can be weakened to the mere existence of $P$. In order to prove this, we shall need the following combinatorial result.

LEмма 3. Let $\mathfrak{C}$ be a denumerable field of sets which contains all finite sets. Suppose further that there is an infinite partition $\boldsymbol{P}_{0}$ of $\boldsymbol{N}$ into infinite sets of $\mathcal{C}$. Then there is an infinite partition $\odot$ of $N$ into infinite sets $P_{0}, P_{1}, \cdots$ of $\mathfrak{C}$ such that for every $S$ in $\mathcal{C}$ either $S$ or $\bar{S}$ is included in a finite union of the $P$ 's.

REMARK. This lemma is stronger than the one I originally proved and was suggested to me by Dana Scott. His proof was obtained by considering the Boolean algebra of sets of $\mathfrak{C}$ mod finite sets.

Proof. Let $S_{0}, S_{1}, \cdots$ be the sets of $\mathcal{C}$. We will define $P_{0}, P_{1}, \ldots$ by means of a sequence of infinite partitions $\mathcal{P}_{0}, \mathcal{P}_{1}, \cdots$ of $N$ into infinite sets of $\mathcal{C}$ such that for every $k$ and $n$ with $k<n, P_{k}$ belongs to $P_{n}$ and either $S_{k}$ or $\bar{S}_{k}$ is included in $\bigcup_{j<n} P_{j}$. Assume that these conditions hold for all $n$ up through $t$. We shall define $P_{t}$ and $P_{t+1}$ so that they also hold for $n=t+1$. Let $S$ be $S_{t}$ if $\bar{S}_{t}$ has infinitely many infinite parts under $\rho_{t}$; otherwise let $S$ be $\bar{S}_{t}$. In either case, $\bar{S}$ has infinitely many infinite parts under $\boldsymbol{P}_{t}$. Let those infinite parts of $\bar{S}$ which are not contained in one of $P_{0}, \cdots, P_{t-1}$ be $I_{0}, I_{1}, \cdots$ Then put 


$$
P_{t}=I_{0} \cup\left(S-\bigcup_{k<t} P_{k}\right)
$$

Let $a_{1}, a_{2}, \cdots$ be the numbers which do not belong to

$$
\bigcup_{k \leqq t} P_{k} \cup \underset{j \geq 1}{\cup} I_{j}
$$

if there are any. Put $T_{k}=I_{k} \cup\left\{a_{k}\right\}$ if there is an $a_{k}$; otherwise let $T_{k}=I_{k}$. Finally, put

$$
\odot_{t+1}=\left\{P_{0}, P_{1}, \cdots, P_{t}, T_{1}, \cdots\right\} .
$$

Clearly $P_{t} \in P_{t+1}, S \subseteq \bigcup_{k \leq t} P_{k}$, and $P_{t+1}$ is an infinite partition of $N$ into infinite sets of $\mathfrak{C}$.

Now let $\odot=\left\{P_{0}, P_{1}, \cdots\right\}$. By construction each $P_{k}$ is an infinite set of $\mathcal{C}, \mathcal{P}$ is disjoint, and for every $k$, either $S_{k}$ or $\bar{S}_{k}$ is contained in $\bigcup_{j \leq k} P_{j}$. Finally, every $n$ belongs to some $P_{j}$. In fact, $\{n\}=S_{l}$ for some $l$, so $n$ belongs to some $P_{j}$ with $j$ not exceeding $l$. Hence $P$ satisfies the lemma.

THEOREM 2. If a denumerable field of sets $\mathcal{e}$ contains all finite sets and includes an infinite disjoint class $\beta$ of infinite sets $P_{0}, P_{1}, \cdots$, then $\mathfrak{C}$ is finitely generated.

Proof. Without loss of generality, we may suppose that $\beta$ is a partition of $\boldsymbol{N}$; otherwise adjoin the missing numbers to the parts $\boldsymbol{P}_{\boldsymbol{k}}$ with at most one to each $P_{k}$. By Lemma 3, we may also assume that $\boldsymbol{P}$ has the further property that for every $S$ in $\mathcal{C}$ either $S$ or $\bar{S}$ is included in a finite union of $P$ 's.

By Lemma 2, we can define a function $H$ so that $H(\boldsymbol{S})=\boldsymbol{P}_{0}$ for every infinite set $S$ in $\mathcal{C}$. Hence $H$ is compatible with $\mathfrak{C}$.

Let $G$ be defined so that $G(S)=P_{n+1}$ for every infinite set $S$ of $\mathfrak{C}$ which is included in $P_{n}$. The existence of $G$ also follows from Lemma 2. Then $G^{k} H(\boldsymbol{N})=\boldsymbol{P}_{k}$ for all $k$. Furthermore, if $\boldsymbol{S}$ is included in a finite union of $P$ 's, then $G(S)$ is the union of a finite set and a finite union of $P$ 's; if $\bar{S}$ is included in a finite union of $P$ 's, then $G(S)$ is the union of a finite set and the complement of a finite union of $P$ 's. In both cases $G(\boldsymbol{S})$ belongs to $\mathrm{e}$. Hence $G$ is compatible with $\mathcal{C}$.

We can now finish the proof by noticing the hypotheses of Theorem 1 hold. We can also construct $F$ more directly as follows: Let $S_{0}$, $S_{1}, \ldots$ be the nonempty sets of $\mathfrak{C}$. By Lemma 2, there is a function $F$ such that $F(T)=S_{k}$ if $T$ is an infinite subset of $P_{k}$ which belongs to e. Then $F$ is compatible with $\mathcal{C}$. Indeed, if $S$ is contained in a finite union of $P$ 's then $F(S)$ is the union of a finite number of sets of $\mathfrak{C}$ and 
if $\overline{\boldsymbol{S}}$ is contained in a finite union of $P$ 's then $F(\boldsymbol{S})=\boldsymbol{N}$. Every nonempty set in $\mathcal{C}$ is generated by $F, G$, and $H$ since $S_{k}=F G^{k} H(N)$.

ThEOREM 3. Let $\mathfrak{C}$ be a denumerable field of sets which contains all finite sets. Suppose $\boldsymbol{A}_{0}, \cdots, \boldsymbol{A}_{n}$ is a partition of $\boldsymbol{N}$ into infinite sets of $\mathfrak{C}$ none of which is the union of two disjoint infinite sets of $\mathfrak{C}$. Then $\mathfrak{C}$ is finitely generated if and only if $n>0$.

Proof. If $n=0$ then $\mathcal{C}$ is the field of all finite and cofinite sets. Suppose $\mathfrak{C}$ were generated by $F_{1}, \cdots, F_{k}, G_{1}, \cdots, G_{l}$ where the range of each $F$ is finite and the range of each $G$ is cofinite. If $G$ is obtained by composition from the $G$ 's then the range of $G$ is cofinite. Hence if $H$ is obtained by composition from the $F$ 's and $G$ 's then $H$ has a finite range only if some $F$, say $F_{j}$, is used to obtain $H$. Then the number of elements in $H(\boldsymbol{N})$ is not more than the number of elements in $F_{j}(N)$. Hence the number of elements in the finite sets generated by $F_{1}, \cdots, F_{k}, G_{1}, \cdots, G_{l}$ is bounded which gives a contradiction. Thus for $n=0, \mathcal{e}$ is not finitely generated.

Suppose $n>0$. For $K \subseteq\{0, \cdots, n\}$, let $S_{K}=\bigcup_{k \in K} A_{k}$. Every set of $e$ differs from some $S_{K}$ by a finite number of elements. We will first show that every $\boldsymbol{S}_{K}$ can be generated by three functions $A, B$, and $C$ which are compatible with $\mathcal{C}$. By Lemma 2, there is a function $A$ such that $A(\boldsymbol{S})=\boldsymbol{A}_{0}$ for every infinite set $\boldsymbol{S}$ in $\mathcal{C}$. $A$ is compatible with $\mathfrak{C}$ since $A(S)$ is either finite or $A_{0}$ for every $S$ in $\mathbb{C}$.

Let $B$ be a permutation which maps $\boldsymbol{A}_{k}$ onto $\boldsymbol{A}_{k+1}$ for $k=0, \cdots$, $n-1$ and maps $A_{n}$ on to $A_{0} . B$ is compatible with $\mathcal{C}$ since $B$ maps any set differing from $S_{K}$ by a finite set onto a set differing from $S_{M}$ by a finite set where $M$ is obtained from $K$ by adding 1 to each element of $K$ other than $n$ and replacing $n$ by 0 . Finally, let $C$ be a function which is one-to-one on each $\boldsymbol{A}_{i}$ and maps $\boldsymbol{A}_{\boldsymbol{i}}$ onto $\boldsymbol{A}_{0} \cup \boldsymbol{A}_{\boldsymbol{i}}$. Again $C$ is compatible with $\mathcal{e}$ since $C$ maps any set differing from $S_{K}$ by a finite set onto a set differing from $S_{M}$ by a finite set where $M=\varnothing$ if $K=\varnothing$ and $M=K \cup\{0\}$ if $K \neq \varnothing$.

Suppose $t$ is the least element of a nonempty set $K \subseteq\{0, \cdots, n\}$. If $K$ has just one element then $S_{K}=B^{t} A(N)$. If $K$ has more than one element, let

$$
K^{\prime}=\{k: k+t \in K \wedge k>0\} .
$$

Then $S_{K}=B^{t} C\left(S_{K^{\prime}}\right)$. Hence by induction on the number of elements of $K$, for every nonempty set $K$, there is a function $H$ obtained from $B$ and $C$ by composition such that $\boldsymbol{S}_{K}=H A(\boldsymbol{N})$.

Now if $S$ differs from $S_{K}$ ( $K$ nonempty) by a finite set, then there is a set $E$ which differs from $\boldsymbol{A}_{0}$ by a finite set such that for some $H$ 
obtained from $B$ and $C$ by composition $H(E)=S$. Indeed, we can choose $H$ as above so that $H\left(A_{0}\right)=S_{K}$; since $H(N)=N$ and $H^{-1}(j)$ is finite for all $j$, we can obtain $E$ by modifying $\boldsymbol{A}_{0}$ by a finite set.

Thus, it remains to show that there are a finite number of functions compatible with $\mathfrak{C}$ which generate every finite set and every set differing from $\boldsymbol{A}_{0}$ by a finite set.

Order the natural numbers in a sequence of the order type of the integers, say $\cdots, a_{-1}, a_{0}, a_{1}, \cdots$, so that $\boldsymbol{A}_{0}=\left\{a_{0}, a_{-1}, a_{-2}, \cdots\right\}$ and for $j>0, a_{j} \in A_{k}$ if $j \equiv k(\bmod n)$ for $k=1, \cdots, n$. Now define three functions $F, G$, and $H$ as follows:

$$
\begin{array}{rlrl}
F\left(a_{k}\right) & =a_{k+1}, & \\
G\left(a_{k}\right)=a_{k} & & \text { if } k \leqq 0, \\
& =a_{k+1} & & \text { if } k>0, \\
H\left(a_{k}\right) & =a_{0} & & \text { if } k \leqq 0, \\
& =a_{k} & & \text { if } k>0 .
\end{array}
$$

We will show that $\mathcal{e}$ is generated by $A, B, C, F, F^{-1}, G$ and $H$. It is easy to check that $F, F^{-1} G$ and $H$ are all compatible with $\mathcal{e}$.

Now for $j, k, l, \cdots$ greater than 0 ,

$$
F\left(\boldsymbol{A}_{0} \cup\left\{a_{j}, a_{k}, a_{l}, \cdots\right\}\right)=\boldsymbol{A}_{0} \cup\left\{a_{1}, a_{j+1}, a_{k+1}, a_{l+1}, \cdots\right\}
$$

and

$$
G\left(A_{0} \cup\left\{a_{j}, a_{k}, a_{l}, \cdots\right\}\right)=A_{0} \cup\left\{a_{j+1}, a_{k+1}, a_{l+1}, \cdots\right\} .
$$

Hence by repeatedly applying $F$ and $G$, we can obtain a function which maps $\boldsymbol{A}_{0}$ onto the union of an arbitrary finite set and $\boldsymbol{A}_{0}$. Next to obtain a set which differs from $A_{0}$ by an arbitrary finite set, we apply a suitable power of $F^{-1}$ to a set which is the union of a finite set and $\boldsymbol{A}_{0}$. On the other hand, if we first apply $H$ to the union of $\boldsymbol{A}_{\mathbf{0}}$ and a finite set and then apply a suitable power of $F$ or $F^{-1}$, we can obtain an arbitrary nonempty finite set.

REMARK. This proof is due to R. M. Robinson. In the original proof, the number of generators depended on $n$.

LEMMA 4. If $\boldsymbol{A}$ can be partitioned into arbitrarily many infinite sets of $\mathfrak{C}$ and $A=B \cup C$ where $B$ and $C$ are infinite sets of $\mathcal{C}$, then at least one of $\boldsymbol{B}$ and $\boldsymbol{C}$ can be partitioned into arbitrarily many infinite sets of $\mathfrak{C}$.

Proof. Let $A$ be partitioned into $2 n$ infinite sets $S_{1}, \cdots, S_{2 n}$ of $\mathfrak{C}$. Then $B=U\left(B \cap S_{k}\right)$ and $C=U\left(C \cap S_{k}\right)$. Either $B$ or $C$ has at least $n$ infinite parts under this partition of $\boldsymbol{A}$ and hence can be partitioned 
into $n$ infinite sets of $\mathfrak{C}$. Hence $B$ or $C$ can be partitioned into arbitrarily many infinite sets of $\mathbb{C}$.

THEOREM 4. If $\mathfrak{e}$ is a denumerable field of sets which contains all finite sets then either $\mathfrak{C}$ is the field of all finite and cofinite sets or $\mathfrak{C}$ is finitely generated.

Proof. Suppose there is a set $\boldsymbol{A}$ in $\mathcal{C}$ such that both $\boldsymbol{A}$ and $\overline{\boldsymbol{A}}$ are infinite. If $N$ can be partitioned into at most $n$ infinite sets of $e$ for some $n$, then Theorem 3 applies. Otherwise $N$ can be partitioned into arbitrarily many infinite sets of $\mathcal{C}$ and by Lemma 4 so can $\boldsymbol{A}$ or $\overline{\boldsymbol{A}}$. Now construct $\boldsymbol{A}_{0}, \boldsymbol{A}_{1}, \cdots$ in turn so that each $\boldsymbol{A}_{j}$ is an infinite set of $\mathfrak{e}$, and $\boldsymbol{N}-\cup_{j \leq k} \boldsymbol{A}_{j}$ can always be partitioned into arbitrarily many infinite sets of $\mathcal{C}$. Of course, we must choose $\boldsymbol{A}_{j}$ to be disjoint from the earlier $A$ 's. Hence Theorem 2 applies and $\mathcal{C}$ is finitely generated.

\section{REFERENCE}

1. Julia Robinson, Finite generation of recursively enumerable sets, Proc. Amer. Math. Soc. 19 (1968), 1480-1486.

University of California, Berkeley 